\title{
Abstract: Understanding challenges stemming from survey on the impact of gender-affirming top-surgery on female- to-male transgender patients
}

Kaitlyn Hines and Megan Lane, MD*

Department of Surgery, University of Michigan Medicine, Ann Arbor, MI 48109

An estimated $0.6 \%$ of the US population is transgender, a term defined as a person whose sense of personal identity does not align with their given natal sex. An increasing number of individuals are presenting to clinics for gender affirming care. For transmen (born natally female), this care often includes chest reconstruction, also referred to as top surgery or mastectomy. Although there is some evidence to suggest gender-affirming treatments improve psychosocial functioning, there is little confirmed research. Already, a challenge of transgender research is the lack thereof. In order to increase the information on the subject, a team worked to collect data on the well-being of transmen after obtaining top-surgery. However, because a limited number of transgender patients are seeking top surgery, another challenge comes to light: obtaining a large enough sample size. Still, the research conducted in this specific survey study aimed to prove that the quality of life increases after top-surgery. The survey collected information from sixty transgender patients and measured patient depression and anxiety rates. It was administered in two parts: the first was given at the first pre-operative appointment, while the second survey is to be given at the 6-month post-operation appointment. Since there is a waiting period between time of consultation, surgery, and post-op appointments, data collecting is still ongoing. As the data is collected, it is entered into an online system known as REDCap. After the complete data has been collected and entered into the system from all sixty patients, we will then analyze the data by comparing the pre-surgery information to the post-surgery data. This work depicts the process and problems which arose along the way when conducting a survey study of transgender health.

^To whom correspondence should be addressed: megalane@med.umich.edu 